\title{
Uma Aplicação da Geometria Analítica em Sistemas de Equações Lineares de Ordem 2 e 3 com Solução Única
}

\section{An Application of Analytical Geometry in Linear Equations Systems of Order 2 and 3 with Unique Solution}

\author{
Adriano Rodrigues de Melo \\ Universidade Federal do Paraná - UFPR, Curitiba, PR \\ melo.a.rodrigues@gmail.com \\ Jair Mendes Marques \\ Universidade Federal do Paraná - UFPR, Curitiba, PR \\ jair.marques@utp.br \\ Renata Burguetti \\ renataburguetti@hotmail.com
}

Resumo: Este artigo propõe uma forma de resolução de sistemas de equações algébricas lineares de ordem 2 e 3 com solução única, baseando-se exclusivamente numa visão geométrica distinta da apresentada em livros de Álgebra Linear. Parte-se do pressuposto que, após algumas operações simples, o produto matricial $A \mathbf{x}, \operatorname{com} A$ de ordem $n \times n$ e $\mathbf{x}$ de ordem $n \times 1$ pode ser reescrito como uma combinação linear das colunas de $A$ e, desse modo, $\mathbf{b}$, de ordem $n \times 1$, é o resultado de uma soma de vetores e, portanto, é a diagonal de um paralelogramo, quando $A$ é de ordem 2 e a diagonal de um prisma trapezoidal, quando $A$ é de ordem 3. A partir desse entendimento, desenvolve-se a proposta.

Palavras-chave: combinação linear; plano; reta; sistemas de equações lineares; vetor.

Abstract: This article proposes a form of a resolution of linear algebraic equation systems of order 2 and 3 with a unique solution, exclusively based on a geometric

Recebido em 08/02/2012 - Aceito em 10/06/2012.

\begin{tabular}{|c|c|c|c|c|c|}
\hline RECEN & 14(1) & p. $135-152$ & jan/jun & 2012 & DOI: $10.5777 /$ RECEN.2012.01.08 \\
\hline
\end{tabular}


view of the linear system presented in separate books of Linear Algebra. It starts with the assumption that after some simple operations, the matrix product $A \mathbf{x}$, with A of order $n \times n$ and $\mathbf{x}$ of order $n \times 1$, can be rewritten as linear a combination of A columns, and in this way, $\mathbf{b}$, of order $n \times 1$, and the result of a sum of vectors and thus is a parallelogram diagonal, and when $\mathrm{A}$ is the order 2 , and the diagonal of a trapezoid prism, and when $\mathrm{A}$ is the order 3 . From that understanding, was developed this proposal.

Key words: linear combination; plan; straight; systems linear equations; vector.

\section{Introdução}

Os sistemas de equações algébricas lineares e suas soluções constituem um dos principais tópicos estudados em cursos conhecidos como "de Álgebra Linear" [1].

Sabe-se que todo sistema de equações lineares tem ou nenhuma solução, ou exatamente uma solução, ou então uma infinidade de soluções [1]. Vários são os métodos para resolver um sistema. Em termos de métodos numéricos, existem os métodos diretos, que são aqueles que fornecem uma resposta em um número fixo de etapas, tais como a eliminação Gaussiana, o método do Gradiente Conjugado, as fatorações LU, LDU, QR, Cholesky e Crout.

Existem, ainda, os métodos iterativos, como os métodos de Gauss-Seidel e GaussJacob que fornecem soluções aproximadas [2].

Para sistemas lineares com solução única, tem-se, também, a Regra de Cramer e a relação $\mathbf{x}=A^{-1} \mathbf{b}$ que, embora sejam ineficientes do ponto de vista computacional, tendo em vista ser muito elevado o número de operações para o cálculo da inversa e do determinante, são muito úteis, pois fornecem uma forma explícita para as soluções de um sistema [3].

Seja um sistema de duas equações em duas incógnitas $x$ e $y$, por exemplo. Dado que os gráficos das equações do sistema são retas, digamos, $l_{1}$ e $l_{2}$, uma interpretação geométrica, comumente encontrada nos livros de Álgebra Linear, para as possibilidades do teorema acima é [1, 3, 4]: 
- As retas $l_{1}$ e $l_{2}$ são paralelas e não têm intersecção, donde não existe solução;

- As retas $l_{1}$ e $l_{2}$ se cruzam (portanto, num único ponto), e existe apenas uma solução;

- As retas $l_{1}$ e $l_{2}$ coincidem, caso em que há uma infinidade de soluções.

Em diversas pesquisas direcionadas ao ensino de Álgebra Linear, de maneira especial, ao tópico de sistemas de equações lineares, o conceito geométrico acima mencionado tem se mostrado de extrema importância e utilidade como caminho para propostas didáticas, produzindo resultados positivamente significativos.

Com efeito, Leivas [5], em sua tese de doutorado, pesquisa a possibilidade do ensino de conceitos geométricos em cursos de Licenciatura Matemática a partir de considerações que envolvam imaginação, intuição e visualização. Neste sentido, o autor salienta a importância da interdisciplinaridade na formação do professor, dizendo que a falta de tal tratamento interdisciplinar ocasiona um conhecimento geométrico limitado, fragmentado e com pouco significado para os futuros professores. No decorrer de seu trabalho, Leivas apresenta inúmeros exemplos de utilização de conceitos geométricos associados às três esferas (imaginação, intuição e visualização) no ensino da Álgebra Linear, Cálculo, Geometria Analítica e até mesmo da Análise.

Em sua dissertação de mestrado, Carneiro [6] constrói, através da geometria vetorial, uma proposta didática para o ensino de sistemas de equações lineares no Ensino Médio. O principal objetivo, segundo o autor, é fornecer, através da geometria vetorial, uma ferramenta que capacite os alunos a analisarem geometricamente sistemas lineares de ordem 3, de modo que o estudo de tal tópico tenha um "maior valor formativo". Para isso, Carneiro salienta a necessidade de incluir, no currículo do Ensino Médio, uma introdução à geometria vetorial.

Jordão [7] desenvolve uma sequência didática que aborda a resolução algébrica e gráfica de sistemas de equações lineares quadrados com o auxílio do software denominado Winplot. A conclusão da pesquisadora aponta para uma relevante importância no uso do referido software, de modo a contribuir para a visualização e compreensão da resolução de sistemas lineares em 3 dimensões. Na mesma esteira e em um nível menos elementar, Rodrigues [8] fornece a descrição e criação de um software 
de apoio ao ensino de base e dimensão de um espaço vetorial, disponibilizando recursos de imagem, vídeo, som e texto. Seus resultados são de melhoria na qualidade do ensino dos referidos tópicos de Álgebra Linear.

Assim, com o fito de contribuir com as pesquisas relativas à interpretação geométrica de sistemas de equações lineares, bem como fornecer uma interação entre áreas da Matemática, o presente artigo tem por objetivo fornecer uma visão geométrica distinta da mencionada acima, tratando sistemas lineares como soma de vetores e, a partir da associação entre alguns conceitos da Geometria Analítica e Álgebra Linear, obter uma forma de resolução geométrica para sistemas possíveis e determinados (SPD) de ordem 2 e 3, procurando explicitar e explorar o potencial da ideia geométrica sugerida.

Então, com a ajuda da Geometria Analítica, é possível dar um novo enfoque ao processo de entendimento e, por sua vez, de resolução de sistemas lineares (ao menos para $n \leq 3$ ), no sentido de mostrar que, geométrica e interdisciplinarmente, são mais sofisticados que a ideia de intersecção dos gráficos das equações que os determinam.

\section{Desenvolvimento}

Seja um sistema de $n$ equações algébricas lineares em $n$ incógnitas na forma:

$$
\left\{\begin{array}{ccc}
a_{11} x_{1}+a_{12} x_{2}+a_{13} x_{3}+\cdots+a_{1 n} x_{n} & = & b_{1} \\
a_{21} x_{1}+a_{22} x_{2}+a_{23} x_{3}+\cdots+a_{2 n} x_{n} & = & b_{2} \\
a_{31} x_{1}+a_{32} x_{2}+a_{33} x_{3}+\cdots+a_{3 n} x_{n} & = & b_{3} \\
\vdots & \vdots & \vdots \\
a_{n 1} x_{1}+a_{n 2} x_{2}+a_{n 3} x_{3}+\cdots+a_{n n} x_{n} & = & b_{n}
\end{array}\right.
$$

em que $b_{i}$ e $a_{i j}$ são números reais para $1 \leq i \leq n$ e $1 \leq j \leq n$, e $i, j \in \mathbb{N}$.

Então, após algumas operações, o sistema de equações lineares $A \mathbf{x}=\mathbf{b}$ (1) pode ser reescrito de forma que o vetor $\mathbf{b}$ seja uma combinação linear das colunas de $A$ 
[1, 3, 4, 9], isto é:

$$
x_{1}\left[\begin{array}{c}
a_{11} \\
a_{21} \\
a_{31} \\
\vdots \\
a_{n 1}
\end{array}\right]+x_{2}\left[\begin{array}{c}
a_{12} \\
a_{22} \\
a_{32} \\
\vdots \\
a_{n 2}
\end{array}\right]+x_{3}\left[\begin{array}{c}
a_{13} \\
a_{23} \\
a_{33} \\
\vdots \\
a_{n 3}
\end{array}\right]+\cdots+x_{n}\left[\begin{array}{c}
a_{1 n} \\
a_{2 n} \\
a_{3 n} \\
\vdots \\
a_{n n}
\end{array}\right]=\left[\begin{array}{c}
b_{1} \\
b_{2} \\
b_{3} \\
\vdots \\
b_{n}
\end{array}\right]
$$

Resolver o sistema (1) consiste em encontrar uma $n$-upla de números (vetor), $\mathbf{x}=$ $\left(x_{1}, x_{2}, \ldots, x_{n}\right) \in \mathbb{R}^{n}$, que satisfaçam simultaneamente as $n$ equações [3]. Para facilitar o desenvolvimento e entendimento, neste artigo, um vetor $\mathbf{u} \in \mathbb{R}^{n}$ será denotado por $\mathbf{u}=\left(u_{1}, u_{2}, \ldots, u_{n}\right)[9]$.

O sistema (1) tem uma única solução se, e somente se, $\operatorname{det} A \neq 0$, o que equivale a dizer que as colunas (linhas) da matriz $A$ são linearmente independentes [1, 3, 4, 9].

Os vetores $\mathbf{v}_{1}, \mathbf{v}_{2}, \cdots, \mathbf{v}_{n}$ são ditos linearmente dependentes (L.D.) se existirem constantes $c_{1}, c_{2}, \cdots, c_{n} \in \mathbb{R}$ nem todas nulas tais que:

$$
c_{1} \mathbf{v}_{1}+c_{2} \mathbf{v}_{2}+\cdots+c_{n} \mathbf{v}_{n}=0
$$

Caso contrário, isto é, se a relação (3) é válida somente se $c_{i}=0$ para todo $\mathrm{i}=1,2$, ..., $\mathrm{n}$ então os vetores $\mathbf{v}_{1}, \mathbf{v}_{2}, \cdots, \mathbf{v}_{n}$ são linearmente independentes (L.I.) [1, 3, 4, 9].

A combinação linear [2] é uma soma de vetores e quando $n=2$, é sabido que a soma de dois vetores $\mathbf{u}$ e $\mathbf{v} \in \mathbb{R}^{2}$, resulta um terceiro vetor $\mathbf{w} \in \mathbb{R}^{2}$, que por sua vez é interpretado como a diagonal de um paralelogramo. Conclusões análogas são válidas para vetores no $\mathbb{R}^{3}$, ou seja, quando $n=3$, casos de interesse uma vez que o "limite geométrico"humano é a terceira dimensão (veja figuras $2 \mathrm{e} 4$ para os casos no $\mathbb{R}^{2}$ e $\mathbb{R}^{3}$, respectivamente).

Este será, então, o modo de se considerarem sistemas lineares (possíveis e determinados): tomando-os simplesmente como soma de vetores. 
Revista Ciências Exatas e Naturais, Vol.14, n 1, Jan/Jun 2012

\subsection{Sistema de 2 equações e 2 incógnitas}

Seja o sistema de equações algébricas lineares $A \mathbf{x}=\mathbf{b}$, tal que $A_{2 \times 2}, \mathbf{x}_{2 \times 1}$ e $\mathbf{b}_{2 \times 1}$ :

$$
\left\{\begin{array}{l}
a_{11} x_{1}+a_{12} x_{2}=b_{1} \\
a_{21} x_{1}+a_{22} x_{2}=b_{2}
\end{array} \Rightarrow\left[\begin{array}{ll}
a_{11} & a_{12} \\
a_{21} & a_{22}
\end{array}\right]\left[\begin{array}{l}
x_{1} \\
x_{2}
\end{array}\right]=\left[\begin{array}{l}
b_{1} \\
b_{2}
\end{array}\right]\right.
$$

Pode-se escrever o vetor $\mathbf{b}$ como uma combinação linear das colunas da matriz $A$ [4]. Assim:

$$
x_{1}\left[\begin{array}{l}
a_{11} \\
a_{21}
\end{array}\right]+x_{2}\left[\begin{array}{l}
a_{12} \\
a_{22}
\end{array}\right]=\left[\begin{array}{l}
b_{1} \\
b_{2}
\end{array}\right] \Rightarrow x_{1} \mathbf{c}_{1}+x_{2} \mathbf{c}_{2}=\mathbf{b}
$$

No caso em que há uma única solução, $\mathbf{c}_{1}$ e $\mathbf{c}_{2}$ são linearmente independentes, o que equivale dizer que um não é combinação linear do outro [1, 3, 4]. Então, fazendo $\mathbf{u}=x_{1} \mathbf{c}_{1}, \mathbf{v}=x_{2} \mathbf{c}_{2}$ e $\mathbf{w}=\mathbf{b}$, em que $\mathbf{c}_{1}=\left(a_{11}, a_{21}\right)$ e $\mathbf{c}_{2}=\left(a_{12}, a_{22}\right)$, o problema de encontrar um vetor $\mathbf{x}=\left(x_{1}, x_{2}\right)$ que satisfaça as duas equações acima [4], é equivalente ao de encontrar o vetor de coeficientes $\mathbf{x}=\left(x_{1}, x_{2}\right)$, que determinam $\mathbf{u}$ e $\mathbf{v}$ cuja soma $\mathbf{u}+\mathbf{v}$ determina $\mathbf{w}=\mathbf{b}$, conforme figura 1 .

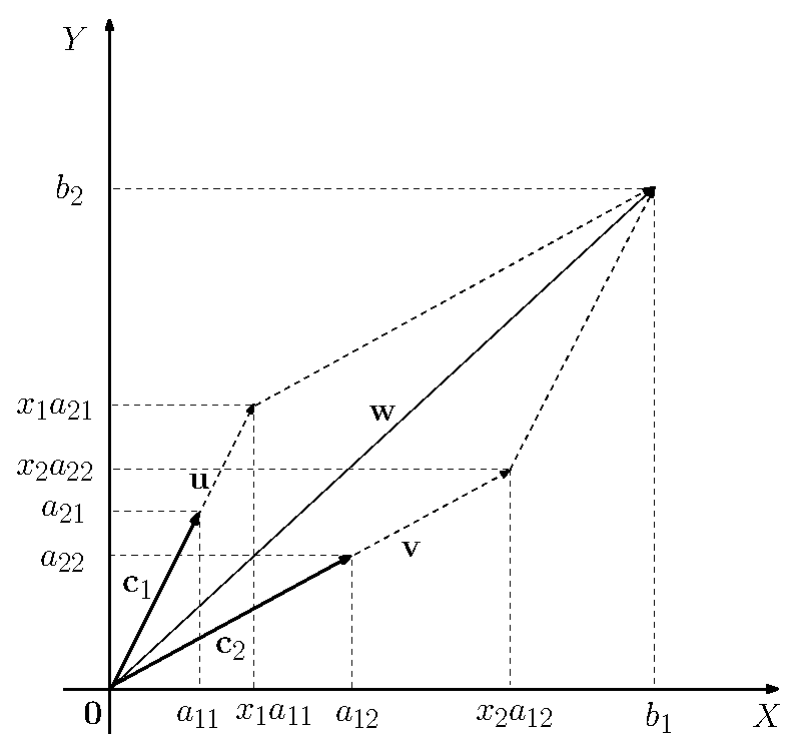

Figura 1. Sistema de ordem 2 como uma soma de vetores 
Da figura 2 é possível observar que:

- O vetor u é múltiplo do vetor $\mathbf{c}_{1}$;

- O vetor v é múltiplo do vetor $\mathrm{c}_{2}$;

- $\mathrm{O}$ vetor $\mathbf{v}$ é determinado pela intersecção das retas $r_{1}$ e $r_{3}$, isto é, $\mathbf{v}=r_{1} \cap r_{3}=$ $\left(x_{2} a_{12}, x_{2} a_{22}\right)$

- O vetor $\mathbf{u}$ é determinado pela intersecção das retas $r_{2}$ e $r_{4}$, isto é, $\mathbf{u}=r_{2} \cap r_{4}=$ $\left(x_{1} a_{11}, x_{1} a_{21}\right)$

- As retas $r_{1}$ e $r_{2}$ são paralelas;

- As retas $r_{3}$ e $r_{4}$ são paralelas;

- $\mathbf{c}_{1}$ e $\mathbf{c}_{2}$ são linearmente independentes (geometricamente, a independência linear entre dois vetores no plano significa que eles não pertencem à uma mesma reta [3]).

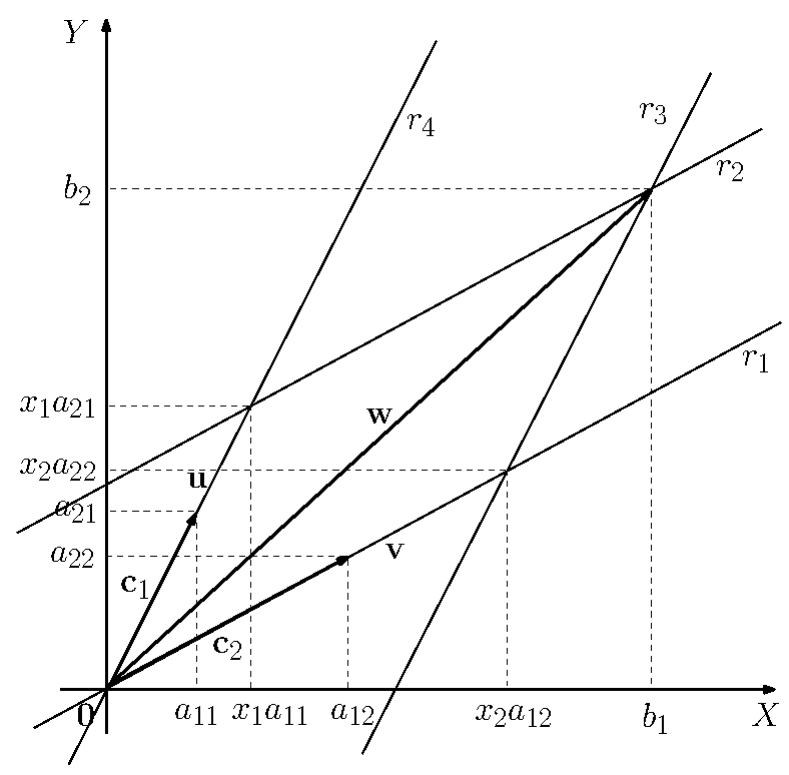

Figura 2. Paralelogramo formado pela soma dos vetores $\mathbf{u} e \mathbf{v}$ 
Então, é possível determinar os vetores $\mathbf{u}=x_{1}\left(a_{11}, a_{21}\right)$ e $\mathbf{v}=x_{2}\left(a_{12}, a_{22}\right)$, e consequentemente os coeficientes $x_{1}$ e $x_{2}$.

\subsubsection{Determinando retas $r_{1}$ e $r_{4}$}

Da figura 2, tem-se que $r_{1}$ passa por $(0,0)$ e $\left(a_{12}, a_{22}\right)$, e, usando a equação da reta $y=c x+d$, implica que:

$$
\left\{\begin{array} { c } 
{ 0 = c \cdot 0 + d } \\
{ a _ { 2 2 } = c \cdot a _ { 1 2 } + d }
\end{array} \Rightarrow \left\{\begin{array}{ccc}
d= & 0 \\
c= & \frac{a_{22}}{a_{12}}
\end{array}\right.\right.
$$

$\operatorname{com} a_{12} \neq 0$. Logo, de $\left[6\right.$ a reta $r_{1}$ pode ser expressa como:

$$
r_{1}: y=\frac{a_{22}}{a_{12}} x
$$

Do mesmo modo, tendo em vista que $r_{4}$ passa por $(0,0)$ e $\left(a_{11}, a_{21}\right)$, conclui-se que a reta $r_{4}$ é dada por:

$$
r_{4}: y=\frac{a_{21}}{a_{11}} x
$$

$\operatorname{para} a_{11} \neq 0$.

\subsubsection{Determinando retas $r_{2}$ e $r_{3}$}

Tem-se, ainda, da figura 2 que $r_{2}$ passa por $\left(b_{1}, b_{2}\right)$ e é paralela à $r_{1}$, isto implica que $r_{2}$ possui o mesmo coeficiente angular da reta $r_{1}$. Então:

$$
r_{2}: y-y_{0}=c\left(x-x_{0}\right) \Rightarrow y=\frac{a_{22}}{a_{12}}\left(x-b_{1}\right)+b_{2}
$$

De maneira análoga, pelo fato de que $r_{3}$ passa pelo ponto $\left(b_{1}, b_{2}\right)$ e é paralela à reta $r_{4}$, tem-se que a reta $r_{3}$ é dada por:

$$
r_{3}: y=\frac{a_{21}}{a_{11}}\left(x-b_{1}\right)+b_{2}
$$




\subsubsection{Determinando vetores $\mathrm{u}$ e $\mathrm{v}$}

Portanto, de posse das quatro equações das retas que delimitam e determinam o paralelogramo formado pela soma dos vetores $\mathbf{u}$ e $\mathbf{v}$ (Figura2) e ainda, lembrando que $\mathbf{v}=r_{1} \cap r_{3}=\left(x_{2} a_{12}, x_{2} a_{22}\right)$, pode-se obter, então, o vetor v. Assim, de (7) e (10):

$$
\begin{aligned}
r_{1} \cap r_{3} & \Rightarrow \frac{a_{22}}{a_{12}} x=\frac{a_{21}}{a_{11}}\left(x-b_{1}\right)+b_{2} \Rightarrow\left(\frac{a_{22}}{a_{12}}-\frac{a_{21}}{a_{11}}\right) x=\frac{a_{11} b_{2}-a_{21} b_{1}}{a_{11}} \\
& \Rightarrow x=a_{12} \cdot \frac{a_{11} b_{2}-a_{21} b_{1}}{a_{11} a_{22}-a_{12} a_{21}}
\end{aligned}
$$

em que $a_{11} a_{22}-a_{12} a_{21} \neq 0$. Substituindo (11) na equação (7):

$$
y=\frac{a_{22}}{a_{12}} x=\left(\frac{a_{22}}{a_{12}}\right) \cdot\left[\frac{a_{12}\left(a_{11} b_{2}-a_{21} b_{1}\right)}{a_{11} a_{22}-a_{12} a_{21}}\right]=a_{22} \cdot \frac{a_{11} b_{2}-a_{21} b_{1}}{a_{11} a_{22}-a_{12} a_{21}}
$$

Então, o vetor $\mathbf{v}$ é dado por:

$$
\mathbf{v}=\left(a_{12} \cdot \frac{a_{11} b_{2}-a_{21} b_{1}}{a_{11} a_{22}-a_{12} a_{21}}, a_{22} \cdot \frac{a_{11} b_{2}-a_{21} b_{1}}{a_{11} a_{22}-a_{12} a_{21}}\right)
$$

De maneira análoga, encontra-se o vetor $\mathbf{u}$ :

$$
\mathbf{u}=\left(a_{11} \cdot \frac{a_{22} b_{1}-a_{12} b_{2}}{a_{11} a_{22}-a_{12} a_{21}}, a_{21} \cdot \frac{a_{22} b_{1}-a_{12} b_{2}}{a_{11} a_{22}-a_{12} a_{21}}\right)
$$

\subsubsection{Determinando $x_{1}$ e $x_{2}$}

Entretanto, como já indicado inicialmente, o interesse nos vetores $\mathbf{u}$ e $\mathbf{v}$ está exclusivamente nos escalares $x_{1}$ e $x_{2}$ que os determinam, conhecidos $\mathbf{c}_{1}, \mathbf{c}_{2}$ e $\mathbf{w}=\mathbf{b}$.

Assim, pelo fato de $\mathbf{u}$ ser múltiplo de $\mathbf{c}_{1}$, de modo que $\mathbf{u}=\left(u_{1}, u_{2}\right)=x_{1}\left(a_{11}, a_{21}\right)$, segue-se que $x_{1}=\frac{u_{1}}{a_{11}}$, ou, equivalentemente, $x_{1}=\frac{u_{2}}{a_{21}}$ se $a_{21} \neq 0$. 
Portanto:

$$
x_{1}=\frac{u_{2}}{a_{21}}=\frac{a_{21}\left(\frac{a_{22} b_{1}-a_{12} b_{2}}{a_{11} a_{22}-a_{12} a_{21}}\right)}{a_{21}}=\frac{a_{22} b_{1}-a_{12} b_{2}}{a_{11} a_{22}-a_{12} a_{21}}=\frac{\left|\begin{array}{ll}
b_{1} & a_{12} \\
b_{2} & a_{22}
\end{array}\right|}{\left|\begin{array}{ll}
a_{11} & a_{12} \\
a_{21} & a_{22}
\end{array}\right|}
$$

Do mesmo modo, $\mathbf{v}$ é múltiplo de $\mathbf{c}_{2}$, implicando, nos mesmos argumentos expostos acima, que $x_{2}=\frac{v_{1}}{a_{12}}$, ou ainda, $x_{2}=\frac{v_{2}}{a_{22}}$ para $a_{22} \neq 0$.

Donde se obtém:

$$
x_{2}=\frac{v_{1}}{a_{12}}=\frac{a_{12}\left(\frac{a_{11} b_{2}-a_{21} b_{1}}{a_{11} a_{22}-a_{12} a_{21}}\right)}{a_{12}}=\frac{a_{11} b_{2}-a_{21} b_{1}}{a_{11} a_{22}-a_{12} a_{21}}=\frac{\left|\begin{array}{ll}
a_{11} & b_{1} \\
a_{21} & b_{2}
\end{array}\right|}{\left|\begin{array}{ll}
a_{11} & a_{12} \\
a_{21} & a_{22}
\end{array}\right|}
$$

Dessa forma, de (14) e 15, tem-se que o procedimento geométrico produz a mesma forma explícita que o método de resolução de equações lineares conhecido como Regra de Cramer, método este utilizado somente no caso de o sistema em questão ser possível e determinado (SPD), ocasião em que a matriz de coeficientes $A$ é invertível (ou não singular).

\subsection{Sistema de 3 equações e 3 incógnitas}

Seja o sistema de equações lineares $A \mathbf{x}=\mathbf{b}, \operatorname{com} A_{3 \times 3}, \mathbf{x}_{3 \times 1}$ e $\mathbf{b}_{3 \times 1}$ :

$$
\left\{\begin{array}{l}
a_{11} x_{1}+a_{12} x_{2}+a_{13} x_{3}=b_{1} \\
a_{21} x_{1}+a_{22} x_{2}+a_{23} x_{3}=b_{2} \\
a_{31} x_{1}+a_{32} x_{2}+a_{33} x_{3}=b_{3}
\end{array} \Rightarrow\left[\begin{array}{lll}
a_{11} & a_{12} & a_{13} \\
a_{21} & a_{22} & a_{23} \\
a_{31} & a_{32} & a_{33}
\end{array}\right]\left[\begin{array}{l}
x_{1} \\
x_{2} \\
x_{3}
\end{array}\right]=\left[\begin{array}{l}
b_{1} \\
b_{2} \\
b_{3}
\end{array}\right]\right.
$$




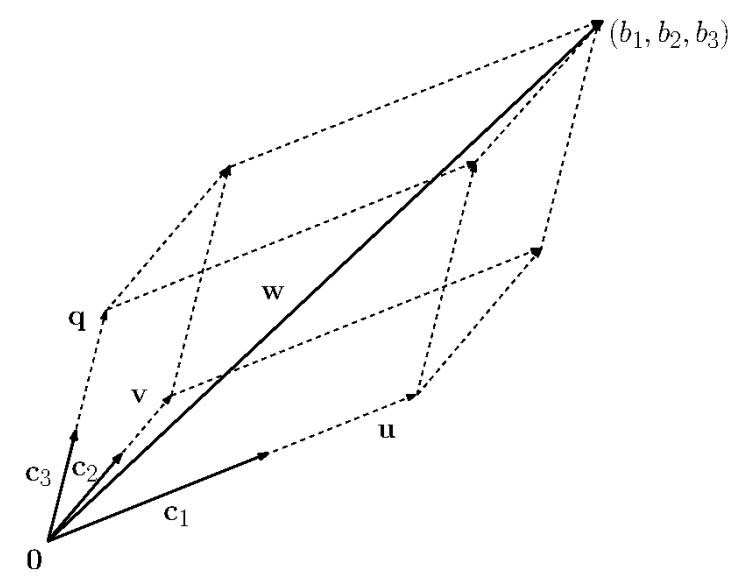

Figura 3. Prisma trapezoidal formado pela soma dos vetores $\mathbf{u}, \mathbf{v}$ e $\mathbf{q}$

Escrevendo, novamente, o vetor $\mathbf{b}=\left(b_{1}, b_{2}, b_{3}\right)$ como uma combinação linear dos vetores colunas $\mathbf{c}_{1}, \mathbf{c}_{2}$ e $\mathbf{c}_{3}$ da matriz de coeficientes $A$ :

$$
x_{1}\left[\begin{array}{l}
a_{11} \\
a_{21} \\
a_{31}
\end{array}\right]+x_{2}\left[\begin{array}{l}
a_{12} \\
a_{22} \\
a_{32}
\end{array}\right]+x_{3}\left[\begin{array}{l}
a_{13} \\
a_{23} \\
a_{33}
\end{array}\right]=\left[\begin{array}{l}
b_{1} \\
b_{2} \\
b_{3}
\end{array}\right] \Rightarrow x_{1} \mathbf{c}_{1}+x_{2} \mathbf{c}_{2}+x_{3} \mathbf{c}_{3}=\mathbf{b}
$$

Como já dito, para que haja solução única $\mathbf{c}_{1}, \mathbf{c}_{2}$ e $\mathbf{c}_{3}$ devem ser L.I. (linearmente independentes). Então, da figura 3, vê-se que o problema de encontrar um vetor solução $\mathbf{x}=\left(x_{1}, x_{2}, x_{3}\right)$ para o sistema de equações lineares (16), é equivalente ao de encontrar o respectivo vetor de coeficientes que determinam os vetores $\mathbf{u}=x_{1} \mathbf{c}_{1}, \mathbf{v}=x_{2} \mathbf{c}_{2}$ e $\mathbf{q}=x_{3} \mathbf{c}_{3}$ que, por sua vez, determinam o vetor $\mathbf{w}$, tal que $\mathbf{w}=\mathbf{b}=\mathbf{u}+\mathbf{v}+\mathbf{q}$.

No desenvolvimento dessas ideias, far-se-á uso de alguns conceitos importantes da Geometria Analítica, como a obtenção das equações paramétricas de uma reta que passa por um ponto e é paralela a um vetor 1 , bem como a determinação da equação geral de um plano definido por três pontos não colineares. Esses conceitos não serão aqui definidos e o leitor interessado poderá consultar as referências [10, 11].

A partir da figura 4 , pode-se observar que:

- O plano $\pi_{1}$ é determinado pelos pontos $\mathrm{O}=(0,0,0), \mathbf{c}_{1}=\left(a_{11}, a_{21}, a_{31}\right)$ e $\mathbf{c}_{3}=$

${ }^{1}$ Optou-se pelas equações paramétricas da reta pelo fato de sua definição no espaço tridimensional ser simples e de fácil aplicação para o presente estudo. 
Revista Ciências Exatas e Naturais, Vol.14, n 1, Jan/Jun 2012

$$
\left(a_{13}, a_{23}, a_{33}\right)
$$

- O plano $\pi_{2}$ é determinado pelos pontos $0=(0,0,0), \mathbf{c}_{1}=\left(a_{11}, a_{21}, a_{31}\right)$ e $\mathbf{c}_{2}=$ $\left(a_{12}, a_{22}, a_{32}\right)$;

- O plano $\pi_{3}$ é determinado pelos pontos $0=(0,0,0), \mathbf{c}_{2}=\left(a_{12}, a_{22}, a_{32}\right)$ e $\mathbf{c}_{3}=$ $\left(a_{13}, a_{23}, a_{33}\right)$;

- A reta $r_{1}$ passa pelo ponto $0=(0,0,0)$ e é paralela ao vetor $\mathbf{c}_{1}$;

- A reta $r_{2}$ passa pelo ponto $0=(0,0,0)$ e é paralela ao vetor $\mathbf{c}_{2}$;

- A reta $r_{3}$ passa pelo ponto $0=(0,0,0)$ e é paralela ao vetor $\mathbf{c}_{3}$;

- Os vetores $\mathbf{c}_{1}, \mathbf{c}_{2}$ e $\mathbf{c}_{3}$ são linearmente independentes (pelo mesmo argumento dado para o caso de vetores no plano, conforme seção 2.1.

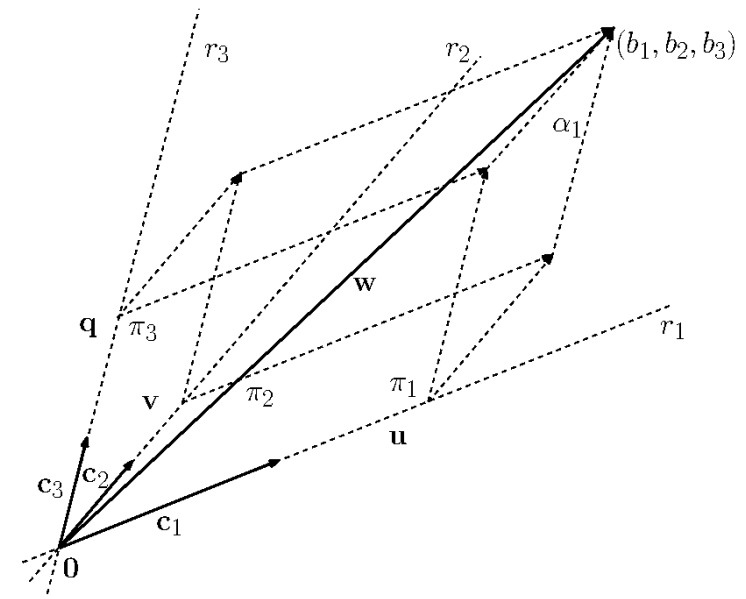

Figura 4. Planos que delimitam o prisma formado pela soma de $\mathbf{u}, \mathbf{v}$ e $\mathbf{q}$ 


\subsubsection{Determinando as equações gerais dos planos}

Pelas informações acima, dados os pontos (Figura 4), podem-se formar os seguintes determinantes e equações gerais dos planos [10, 11]:

$$
\begin{gathered}
\pi_{1}:\left|\begin{array}{ccc}
x-0 & y-0 & z-0 \\
a_{11}-0 & a_{21}-0 & a_{31}-0 \\
a_{13}-0 & a_{23}-0 & a_{33}-0
\end{array}\right|=0 \\
\Rightarrow \pi_{1}:\left(a_{21} a_{33}-a_{31} a_{23}\right) x+\left(a_{31} a_{13}-a_{11} a_{33}\right) y+\left(a_{11} a_{23}-a_{21} a_{13}\right) z=0 \\
\pi_{2}:\left|\begin{array}{ccc}
x-0 & y-0 & z-0 \\
a_{12}-0 & a_{22}-0 & a_{32}-0
\end{array}\right|=0 \\
\Rightarrow \pi_{2}:\left(a_{21} a_{32}-a_{31} a_{22}\right) x+\left(a_{31} a_{12}-a_{11} a_{32}\right) y+\left(a_{11} a_{22}-a_{21} a_{12}\right) z=0 \\
\pi_{3}:\left|\begin{array}{ccc}
x-0 & y-0 & z-0 \\
a_{12}-0 & a_{22}-0 & a_{32}-0 \\
a_{13}-0 & a_{23}-0 & a_{33}-0
\end{array}\right|=0 \\
\Rightarrow \pi_{3}:\left(a_{22} a_{33}-a_{32} a_{23}\right) x+\left(a_{32} a_{13}-a_{12} a_{33}\right) y+\left(a_{12} a_{23}-a_{22} a_{13}\right) z=0
\end{gathered}
$$

O plano $\alpha_{1}$ (Figura 4 ) é paralelo ao plano $\pi_{3}$ e passa pelo ponto $\left(b_{1}, b_{2}, b_{3}\right)$. Sabe-se que dois planos:

$$
\beta_{1}: a_{1} x+b_{1} y+c_{1} z=e_{1} \quad \beta_{2}: a_{2} x+b_{2} y+c_{2} z=e_{2}
$$

são paralelos se, e somente se, existe $k \in \mathbb{R}$ tal que $\frac{a_{1}}{a_{2}}=\frac{b_{1}}{b_{2}}=\frac{c_{1}}{c_{2}}=k[10$, 11]. Logo, deve existir $k_{1} \in \mathbb{R}$, de modo que, pela equação geral do plano $\pi_{3}$ acima:

$$
\alpha_{1}: k_{1}\left(a_{22} a_{33}-a_{32} a_{23}\right) x+k_{1}\left(a_{32} a_{13}-a_{12} a_{33}\right) y+k_{1}\left(a_{12} a_{23}-a_{22} a_{13}\right) z=d_{1}
$$


Revista Ciências Exatas e Naturais, Vol.14, n 1, Jan/Jun 2012

E, substituindo o ponto $\left(b_{1}, b_{2}, b_{3}\right)$ nesta equação $[18)$, tem-se:

$$
d_{1}=k_{1}\left(a_{22} a_{33}-a_{32} a_{23}\right) b_{1}+k_{1}\left(a_{32} a_{13}-a_{12} a_{33}\right) b_{2}+k_{1}\left(a_{12} a_{23}-a_{22} a_{13}\right) b_{3}
$$

Logo, por (18) e (19), a equação geral do plano $\alpha_{1}$ está completamente determinada.

\subsubsection{Determinando as equações paramétricas das retas}

Das informações organizadas na seção 2.2, as equações paramétricas das retas $r_{1}$, $r_{2}$ e $r_{3}$ são [10,11]:

$$
r_{1}:\left\{\begin{array}{rl}
x & =a_{11} t_{1} \\
y & =a_{21} t_{1} \\
z & =a_{31} t_{1}
\end{array} \quad r_{2}:\left\{\begin{array}{rl}
x & =a_{12} t_{2} \\
y & =a_{22} t_{2} \\
z & =a_{32} t_{2}
\end{array} \quad r_{3}:\left\{\begin{array}{l}
x=a_{13} t_{3} \\
y=a_{23} t_{3} \\
z=a_{33} t_{3}
\end{array}\right.\right.\right.
$$

\subsubsection{Determinando $x_{1}, x_{2}$ e $x_{3}$}

Da figura 4 , observa-se que o vetor $\mathbf{u}=x_{1} \mathbf{c}_{1}$ pode ser determinado pela intersecção do plano $\alpha_{1}$ com a reta $r_{1}$, isto é: $\mathbf{u}=\alpha_{1} \cap r_{1}$. Assim, para obter o vetor $\mathbf{u}$ basta determinar o ponto dado pela referida intersecção.

Para tanto, substituindo as equações paramétricas da reta $r_{1} \mathrm{em}$ na equação do plano $\alpha_{1}$ dada por $\left[18\right.$ e pondo em evidência o termo $k_{1} t_{1}$, obtém-se [10]:

$$
k_{1} t_{1}=\frac{d_{1}}{a_{11} a_{22} a_{33}-a_{11} a_{32} a_{23}+a_{21} a_{32} a_{13}-a_{12} a_{21} a_{33}+a_{31} a_{12} a_{23}-a_{31} a_{22} a_{13}}
$$

$\operatorname{com} a_{11} a_{22} a_{33}-a_{11} a_{32} a_{23}+a_{21} a_{32} a_{13}-a_{12} a_{21} a_{33}+a_{31} a_{12} a_{23}-a_{31} a_{22} a_{13} \neq 0$.

Agora, substituindo $d_{1}$ dado por 19 na equação anterior, com $k_{1} \neq 0$, resulta em:

$$
t_{1}=\frac{b_{1} a_{22} a_{33}-b_{1} a_{32} a_{23} b_{1}+b_{2} a_{32} a_{13}-b_{2} a_{12} a_{33}+b_{3} a_{12} a_{23}-b_{3} a_{22} a_{13}}{a_{11} a_{22} a_{33}-a_{11} a_{32} a_{23}+a_{21} a_{32} a_{13}-a_{12} a_{21} a_{33}+a_{31} a_{12} a_{23}-a_{31} a_{22} a_{13}}
$$


Então, dado $t_{1}$ acima, tem-se:

$$
\mathbf{u}=x_{1} \mathbf{c}_{1} \Rightarrow\left(a_{11} t_{1}, a_{21} t_{1}, a_{31} t_{1}\right)=\left(x_{1} a_{11}, x_{1} a_{21}, x_{1} a_{31}\right) \Leftrightarrow x_{1}=t_{1}
$$

Logo:

$$
x_{1}=\frac{b_{1} a_{22} a_{33}-b_{1} a_{32} a_{23} b_{1}+b_{2} a_{32} a_{13}-b_{2} a_{12} a_{33}+b_{3} a_{12} a_{23}-b_{3} a_{22} a_{13}}{a_{11} a_{22} a_{33}-a_{11} a_{32} a_{23}+a_{21} a_{32} a_{13}-a_{12} a_{21} a_{33}+a_{31} a_{12} a_{23}-a_{31} a_{22} a_{13}}
$$

Que, por outro lado pode ser expresso como:

$$
x_{1}=\frac{\left|\begin{array}{lll}
b_{1} & a_{12} & a_{13} \\
b_{2} & a_{22} & a_{23} \\
b_{3} & a_{32} & a_{33}
\end{array}\right|}{\left|\begin{array}{lll}
a_{11} & a_{12} & a_{13} \\
a_{21} & a_{22} & a_{23} \\
a_{31} & a_{32} & a_{33}
\end{array}\right|}
$$

De maneira análoga, considerando o plano $\alpha_{2}$ como aquele que passa por $\left(b_{1}, b_{2}, b_{3}\right)$ e é paralelo ao plano $\pi_{3}$, segue-se que o vetor $\mathbf{v}$ é dado pela intersecção da reta $r_{2}$ com $\alpha_{2}$ (Figura 4), de modo que, pelo mesmo argumento dado acima, é possível determinar $x_{2}$ como sendo:

$$
x_{2}=\frac{\left|\begin{array}{lll}
a_{11} & b_{1} & a_{13} \\
a_{21} & b_{2} & a_{23} \\
a_{31} & b_{3} & a_{33}
\end{array}\right|}{\left|\begin{array}{lll}
a_{11} & a_{12} & a_{13} \\
a_{21} & a_{22} & a_{23} \\
a_{31} & a_{32} & a_{33}
\end{array}\right|}
$$

Por fim, da mesma maneira encontra-se $x_{3}$, uma vez que q é a intersecção da reta $r_{3}$ com o plano $\alpha_{3}$ que passa, também, por $\left(b_{1}, b_{2}, b_{3}\right)$ e é paralelo ao plano $\pi_{2}$ (Figura 
4). Donde se tem:

$$
x_{3}=\frac{\left|\begin{array}{lll}
a_{11} & a_{12} & b_{1} \\
a_{21} & a_{22} & b_{2} \\
a_{31} & a_{32} & b_{3}
\end{array}\right|}{\left|\begin{array}{lll}
a_{11} & a_{12} & a_{13} \\
a_{21} & a_{22} & a_{23} \\
a_{31} & a_{32} & a_{33}
\end{array}\right|}
$$

Logo, das igualdades (21), 22) e 23], segue-se que o procedimento geométrico para a resolução do sistema de equações lineares de ordem três fornece a mesma forma explícita que o método de Cramer para sistemas possíveis e determinados (solução única).

\subsection{Sistema de $n$ equações e $n$ incógnitas}

Como já visto na seção 2, o sistema linear (1) de $n$ equações em $n$ incógnitas pode ser transformado em 44 , de modo que $\mathbf{b}=\sum_{i=1}^{n} x_{i} \mathbf{c}_{i}, i=1,2, \ldots, n$. Uma vez que estão sendo considerados sistemas possíveis e determinados (SPD), as colunas $\mathbf{c}_{i}$, para todo $i$, são linearmente independentes e dado qualquer $\mathbf{b}$, existe um único vetor $\mathbf{x}$, tal que $A \mathbf{x}=\mathbf{b}$ [1, 3, 4, 9]. Assim, embora $\mathbf{b}$ pertença ao espaço coluna de $A$, não é possível aplicar o raciocínio que vem sendo desenvolvido neste trabalho para $n \geq 4$ tendo em vista, conforme já mencionado, a capacidade de abstração visual humana não exceder à terceira dimensão.

\section{Conclusões}

Neste artigo, um sistema de equações lineares com solução única foi tomado analítica e geometricamente como uma soma de vetores. Assim, apresentaram-se sistemas lineares de ordem 2 e 3 que foram resolvidos, de forma genérica, por um procedimento geométrico. Este procedimento foi conduzido, principalmente, com os conceitos de independência e combinação linear da Álgebra Linear, bem como de vetor, plano e reta da Geometria Analítica, de modo que, tomando um sistema linear 
como uma soma de vetores, que produz uma série de hiperplanos que se interceptam, é possível obter a solução do sistema analisando simplesmente as suas intersecções. Ao final, obtemos a solução exata dos sistemas de ordem 2 e 3 , de modo que a forma explícita da solução foi equivalente aquela fornecida pela Regra de Cramer.

Concluímos, então, que houve eficácia no desenvolvimento das ideias no que tange a formular uma interpretação geométrica para sistemas lineares distinta da apresentada em livros de Álgebra Linear, bem como alcançar um procedimento de resolução geométrico, para sistemas de ordem 2 e 3, que explorasse as características subjacentes à interpretação supracitada, pois tratou-se de uma abordagem baseada nos aspectos geométricos propostos e equivalente, em termos de resultado, ao método de Cramer.

Concluímos ademais que, do ponto de vista pedagógico, a abordagem apresentada é de grande importância e utilidade prática interdisciplinar, pois possui o caráter de contribuir na aplicação, fixação e conservação dos conceitos de Álgebra Linear e Geometria Analítica, uma vez que abrange várias ideias destas áreas que são trabalhadas num curso regular.

Não obstante, outras propostas de nível pedagógico podem ser formadas a partir do contexto geométrico estudado, como por exemplo, a análise do volume formado pelos sólidos tratados e sua relação com a solução de sistemas possíveis e determinados (SPD).

Saliente-se, por fim, que sua principal contribuição é sua simplicidade de organização e inovação (observe que o procedimento de resolução foi estruturado e conduzido pelas colunas da matriz de coeficientes $A$, ao contrário dos métodos tradicionais), pois os conceitos utilizados representam ferramentas básicas da Geometria Analítica e da Álgebra Linear.

\section{Referências}

[1] ANTON, R.; RORRES, C. Álgebra Linear com Aplicações. Ed. Bookman, $8^{a}$ ed., Porto Alegre, 2001.

[2] BURDEN, R. L.; FAIRES, J. D. Análise Numérica. Ed. Cengage Learning, $8^{a}$ 
ed., São Paulo, 2008.

[3] BOLDRINI, J. L.; COSTA, S. I. R.; FIGUEIREDO, V. L.; WETZLER, H. G. Álgebra Linear. Ed. Harbra Ltda, $3^{a}$ ed., São Paulo, 1980.

[4] KOLMAN, B.; HILL, D. R. Introdução à Álgebra Linear com Aplicações. Ed. LTC, $2^{a}$ ed., Rio de Janeiro, 2006.

[5] LEIVAS, J. C. P. Imaginação, Intuição e Visualização: A Riqueza de Possibilidades da Abordagem Geométrica no Currículo de Cursos de Licenciatura de Matemática. Tese de Doutorado, Universidade Federal do Paraná. Curitiba, 2009.

[6] CARNEIRO, P. S. Geometria Vetorial na Escola: Uma Leitura Geométrica para Sistemas de Equações. Dissertação de Mestrado, Universidade Federal do Rio Grande do Sul. Instituto de Matemática, Porto Alegre, 2007.

[7] JORDÃO, A. L. I. Um Estudo sobre a resolução algébrica e gráfica de Sistemas Lineares $3 \times 3$ no $2^{\circ}$ ano do Ensino Médio. Dissertação de Mestrado, Pontifícia Universidade Católica de São Paulo. São Paulo, 2011.

[8] RODRIGUES, J. R. F. Criação de Um Software de Apoio ao Ensino e à Aprendizagem de Álgebra Linear. Dissertação de Mestrado, Pontifícia Universidade Católica de Minas Gerais. Belo Horizonte, 2009.

[9] LIMA, E. L. Álgebra Linear. IMPA - Instituto de Matemática Pura e Aplicada, CNPq, $8^{a}$ ed., Rio de Janeiro, 2011.

[10] VENTURI, J. J. Álgebra Vetorial e Geometria Analítica. $9^{a}$ ed., Curitiba.

[11] STEINBRUCH, A., WINTERLE, P. Geometria Analítica. Ed. Person Makron Books Editora LTDA, $2^{a}$ ed., São Paulo, 1987. 